\title{
Anti-inflammatory effects of Panax notoginseng saponins ameliorate acute lung injury induced by oleic acid and lipopolysaccharide in rats
}

\author{
YU-QING CHEN $^{1}$, LING RONG ${ }^{1}$ and JIAN-OU QIAO ${ }^{2}$ \\ ${ }^{1}$ Department of Respiratory Medicine, Shanghai First People's Hospital, Shanghai Jiaotong University School of Medicine, \\ Shanghai 200080; ${ }^{2}$ Department of Respiratory Medicine, Shanghai Ninth People's Hospital, \\ Shanghai Jiaotong University School of Medicine, Shanghai 200011, P.R. China
}

Received July 16, 2013; Accepted January 22, 2014

DOI: $10.3892 / \mathrm{mmr} .2014 .2328$

\begin{abstract}
This study investigated the effect of Panax notoginseng saponins (PNS) on acute lung injury (ALI) induced by oleic acid (OA) and lipopolysaccharide (LPS). A total of 28 Wistar rats were divided into four groups: sham; sham + PNS; OA-LPS-induced ALI and ALI + PNS. Lung tissue histology, lung wet-to-dry (W/D) weight ratio, extravascular lung water $($ EVLW) and epithelial sodium channel $\alpha(\alpha E N a C)$ mRNA and protein expression were examined. In addition, levels of inflammatory cytokines, including tumor necrosis factor $\alpha$ (TNF- $\alpha$ ), interleukin (IL)-6 and IL-10, as well as total leukocyte and neutrophil counts, were analyzed in rat bronchoalveolar lavage fluid (BALF) and serum. ALI + PNS rats were observed to exhibit significantly lower pulmonary parenchymal damage and EVLW compared with ALI rats. Furthermore, total leukocyte and neutrophil counts, and levels of inflammatory cytokines were significantly decreased following PNS administration in ALI rats. In addition, the decrease in $\alpha \mathrm{ENaC}$ mRNA and protein expression observed in the lung tissue of ALI rats was partially restored following PNS treatment. PNS treatment was demonstrated to ameliorate OA-LPS-induced ALI, potentially through restoration of $\alpha \mathrm{ENaC}$ mRNA and protein expression and through PNS-induced anti-inflammatory effects.
\end{abstract}

\section{Introduction}

Acute respiratory distress syndrome (ARDS) is characterized by acute respiratory failure resulting from acute lung

Correspondence to: Dr Jian-Ou Qiao, Department of Respiratory Medicine, Shanghai Ninth People's Hospital, Shanghai Jiaotong University School of Medicine, 639 Zhizaoju Road, Shanghai 200011, P.R. China

E-mail: qiaojianou@yeah.net

Key words: acute lung injury, alveolar liquid clearance, epithelial sodium channel, extravascular lung water, Panax notoginseng saponins injury (ALI). The main characteristics of ARDS are diffuse inflammation and increased microvascular permeability that cause interstitial and alveolar edema and persistent refractory hypoxemia (1). While ALI may be caused by various insults, it has been suggested that a common pathway is likely to result in lung damage (2-4).

In vivo alveolar fluid volume is determined by alveolar fluid clearance (AFC), which is a function of transepithelial $\mathrm{Na}^{+}$transport (5). AFC has been reported to be impaired in ARDS/ALI and has been associated with the function of epithelial sodium channels (ENaCs) (6). ENaCs are composed of three homologous subunits, $\alpha, \beta$ and $\gamma$ (7). In a lipopolysaccharide (LPS)-induced model of ALI in rats, all three ENaC subunits were observed to be downregulated in lung epithelial tissue at the protein and mRNA levels. This was concomitant with LPS-induced ALI (8).

It is well established that LPS is capable of inducing inflammatory responses and that ALI involves a complex series of inflammatory events that result in increased alveolar-capillary membrane permeability (9-11). Numerous cytokines are produced in the lung by resident cells, including alveolar macrophages, lung epithelial cells and fibroblasts, or by neutrophils, lymphocytes, monocytes and platelets in response to local or systemic injury (12-16). Inflammatory cytokines, including tumor necrosis factor $\alpha$ (TNF- $\alpha)$, interleukin (IL)-6 and IL-8, have been reported to have a critical role in the pathophysiology of septic shock, a condition that frequently leads to ALI (17). It has been hypothesized that ineffective lung repair following ALI is a consequence of persistent inflammatory stimulation (18).

Panax notoginseng saponins (PNS) are extracts of the Chinese herb, $P$. notoginseng. The major active components of PNS are ginsenoside Rb1 (Rb1), ginsenoside Rg1 (Rg1) and notoginsenoside R1 (R1), which have been identified to exhibit a variety of pharmacological activities, including anti-inflammatory and antioxidant effects, in addition to calcium antagonist activities. It has been reported that PNS may protect against cerebral ischemia and exert beneficial effects on the cardiovascular system, as well as possess hemostatic, antioxidant and estrogen-like activities (19-21). We previously demonstrated that PNS $(100 \mathrm{mg} / \mathrm{kg})$ treatment was capable 
of alleviating intestinal ischemia/reperfusion (II/R)-induced ALI in rats and that it significantly reduced oxidant enzyme activity, levels of malondialdehyde (MDA) and nitric oxide, the activity of inducible nitric oxide synthase in lung tissue and pro-inflammatory cytokine levels in the plasma (22).

The present study investigated the protective effects of PNS on ALI induced in a rat model. ALI was induced using oleic acid (OA) and LPS (23). OA has been reported to inhibit alveolar fluid reabsorption, which is a significant problem in ARDS (24). The endotoxin, LPS, has also been reported to have adverse effects on lung tissue water homeostasis, as it adversely affects ENaC-mediated $\mathrm{Na}^{+}$transport $(25,26)$. This study analyzed the effects of PNS on lung tissue histology, extravascular lung water (EVLW) levels and lung tissue $\alpha \mathrm{ENaC}$ mRNA and protein expression. In order to assess the anti-inflammatory properties of PNS, inflammatory cytokines, including TNF- $\alpha$, IL- 6 and IL-10, were examined in the serum and bronchoalveolar lavage fluid (BALF) of the rats. These are the predominant cytokines associated with ALI in animal models (23).

\section{Materials and methods}

Animals. Healthy male Wistar rats weighing 200-250g were purchased from the Shanghai Laboratory Animal Centre (SLAC; Shanghai, China). The rats were randomly assigned to experimental groups and housed in groups of four under environmentally controlled conditions in compliance with the Policy on Animal Care and Use, Shanghai Jiaotong University (Shanghai, China). All experiments were approved by the Ethics Committee of the Faculty of Pharmacy, Shanghai Jiaotong University.

Rats used for the model of OA and LPS-induced ALI were fasted and permitted solely water for $24 \mathrm{~h}$ prior to commencing the experiment. Rats were anesthetized using $10 \%$ chloral hydrate $(0.35 \mathrm{ml} / \mathrm{kg})$ and heparin $(400 \mathrm{U} / \mathrm{kg})$ administered intraperitoneally. A 4-Fr double-lumen catheter (Arrow International, Reading, PA, USA) was inserted through the jugular vein for fluid and drug infusion. Anesthesia was maintained using ketamine at $1 \mathrm{mg} / \mathrm{kg} / \mathrm{h}$ and pancuronium at $0.3 \mathrm{mg} / \mathrm{kg} / \mathrm{h}$ through the central venous line. Lactated Ringer's solution was infused $(10 \mathrm{ml} / \mathrm{kg} / \mathrm{h})$ throughout the procedure.

An arterial catheter was placed in the carotid artery to monitor the arterial pressure and to sample the arterial blood. A heating pad was used to maintain body temperature at $\sim 37^{\circ} \mathrm{C}$. Rats were placed in the supine position and a midline cervical incision was performed followed by tracheostomy, whereby the trachea was intubated with a $2.0-\mathrm{mm}$-diameter tracheal tube. Mechanical ventilation was performed using an animal ventilator (Model ALC-V8S; Shanghai Alcott Biotech Co. Ltd., Shanghai, China) as follows: tidal volume $=15 \mathrm{ml} / \mathrm{kg}$; respiratory rate $(\mathrm{RR})=40$ breaths/min; inspiratory:expiratory ratio $=1: 1$; fraction of inspiratory oxygen $\left(\mathrm{FiO}_{2}\right)=0.21$.

Experimental protocols. A total of 28 rats were randomly assigned to four groups of seven rats: a sham group (group 1), a sham + PNS group (group 2), an ALI group (group 3) and an ALI + PNS group (group 4). Rats in group 1 were anesthetized and received intravenous (IV) saline $(2 \mathrm{ml} / \mathrm{kg})$, solely. Rats in group 2 were also administered IV saline as well as
PNS (100 mg/kg; Yunnan Phytopharmaceutical Co. Ltd., Kunming, China) by bolus IV injection. PNS was prepared in a $5 \%$ glucose solution with sterile water. Rats in group 3 received IV saline $(2 \mathrm{ml} / \mathrm{kg})$ and an IV injection of OA at $0.2 \mathrm{ml} / \mathrm{kg}$ (Sigma-Aldrich, St. Louis, MO, USA) followed by $4 \mathrm{~h}$ of LPS injection $(5 \mathrm{mg} / \mathrm{kg})$. Rats in group 4 were administered PNS (100 mg/kg) following ALI stabilization for $15 \mathrm{~min}$. At $2 \mathrm{hr}$ subsequent to these treatments, rats were sacrificed by cardiac puncture under anesthesia induced by intramuscular injection with ketamine and xylazine at 8.7 and $1.3 \mathrm{mg} / 100 \mathrm{~g}$ body weight (BW), respectively. Samples were taken for the analyses described below.

Lung histology. The right lower lobes of the lungs were harvested and fixed in $10 \%$ formalin. After $48 \mathrm{~h}$, the tissue samples were embedded in paraffin. Paraffin-embedded, 4-mm-thick sections were stained using hematoxylin and eosin (H\&E) and examined using light microscopy with an attached photo-documentation device (Carl Zeiss Shanghai Co., Ltd., Shanghai, China).

Lung wet-to-dry (W/D) weight ratio and EVLW. Pulmonary edema was determined by weighing both lungs at necropsy and then drying the tissue at $70^{\circ} \mathrm{C}$ until the dry weight remained unchanged for $24 \mathrm{~h}$. The lung W/D weight ratio was normalized to the initial body weight, as was EVLW, as described previously (27). In brief, equal quantities of lung tissue and distilled water were homogenized and a total of $50 \mathrm{ml}$ homogenate was centrifuged at 2,000 $\mathrm{xg}$ (centrifuge radius $=17.4 \mathrm{~cm}$ ) for $10 \mathrm{~min}$, prior to incubation at $5^{\circ} \mathrm{C}$ for $1 \mathrm{~h}$. The hemoglobin ( $\mathrm{Hb}$ ) concentration was measured in the supernatant. Samples of arterial blood, tissue homogenates and supernatants were dried at $80^{\circ} \mathrm{C}$ for $72 \mathrm{~h}$ and the water content percentages of the samples were calculated. The calculations were performed using the following formulae: Homogenate $\mathrm{Hb}$ concentration = supernatant $\mathrm{Hb}$ x (homogenate water content \%/supernatant water content \%); blood weight $=$ homogenate weight $\mathrm{x}$ (homogenate $\mathrm{Hb} /$ blood $\mathrm{Hb}$ ); blood water weight $=$ blood weight $\mathrm{x}$ blood water content $\%$; lung water content $(\mathrm{TPW})=$ (homogenate water content $\%$ x homogenate weight) - volume of distilled water added; EVLW = TPW - blood water weight.

$B A L F$ collection and cell counts. The right main bronchus was clamped and BAL of the left lung was performed three times using $2 \mathrm{ml}$ saline solution. The cell suspension was centrifuged at $100 \mathrm{xg}$ for $10 \mathrm{~min}$ at $4^{\circ} \mathrm{C}$, following which cells were resuspended in $1 \mathrm{ml}$ saline solution. Glass slides were coated with $0.1 \mathrm{ml}$ cell suspension, prior to staining with Wright-Giemsa. A total of 400 nucleated cells were examined using light microscopy to calculate total and differential cell counts.

Cytokine ELISAs. Prior to sacrifice, blood samples were obtained from the abdominal aorta and centrifuged to isolate the sera, which were stored at $-80^{\circ} \mathrm{C}$. TNF- $\alpha$, IL- 6 and IL-10 levels were quantified using a Bio-Plex Rat Serum Diluent Kit (Bio-Rad Laboratories, Inc., Hercules, CA, USA) according to a modified double-ligand method described previously (28). Flat-bottomed, 96-well microtiter plates were coated with $50 \mathrm{ml} /$ well rat antibody against the various cytokines at a 
concentration of $1 \mathrm{mg} / \mathrm{ml}$ in $0.6 \mathrm{M} \mathrm{NaCl}, 0.26 \mathrm{M} \mathrm{H}_{3} \mathrm{BO}_{4}$ and $0.08 \mathrm{M} \mathrm{NaOH}(\mathrm{pH} 9.6)$ for $16 \mathrm{~h}$ at $4^{\circ} \mathrm{C}$. Samples were then washed with phosphate-buffered saline (PBS; pH 7.5) containing $0.05 \%$ Tween 20 (wash buffer). Non-specific binding sites were blocked using $2 \%$ bovine serum albumin (BSA) in PBS and incubated for $90 \mathrm{~min}$ at $37^{\circ} \mathrm{C}$. Plates were then rinsed four times with wash buffer, and $50 \mu \mathrm{l}$ neat and 1:10 diluted cell-free supernatants were added to duplicate wells and incubated for $1 \mathrm{~h}$ at $37^{\circ} \mathrm{C}$. Plates were washed four times, prior to the addition of $50 \mu \mathrm{l} /$ well biotinylated rabbit antibodies against the specific cytokines at a concentration of $3.5 \mathrm{mg} / \mathrm{ml}$ in wash buffer containing $2 \%$ FCS. Plates were then incubated for $30 \mathrm{~min}$ at $37^{\circ} \mathrm{C}$, prior to four washes and incubation with streptavidin-peroxidase conjugate (Bio-Rad Laboratories, Inc.) for $30 \mathrm{~min}$ at $37^{\circ} \mathrm{C}$. Plates were then washed a further four times, followed by the addition of chromogen substrate (Bio-Rad Laboratories, Inc.). Plates were incubated at room temperature and the reaction was terminated with $50 \mu \mathrm{l} /$ well $3 \mathrm{M} \mathrm{H}_{2} \mathrm{SO}_{4}$ solution. Plates were read at $490 \mathrm{~nm}$ using an ELISA reader.

Standards were $1 / 2 \log$ dilutions of recombinant murine cytokines from $1 \mathrm{pg} / \mathrm{ml}$ to $100 \mathrm{ng} / \mathrm{ml}$. This ELISA method consistently detectedmurinecytokine concentrations $>25 \mathrm{pg} / \mathrm{ml}$. These ELISAs were not detected to cross-react with IL-1, IL-2 or IL-4, or with members of the murine chemokine family, including murine $\mathrm{JE} /$ monocyte chemoattractant protein (MCP)-1, regulated on activation, normal T cell expressed and secreted (RANTES), keratinocyte-derived chemokine (KC), macrophage inflammatory protein (MIP)-2, growth-related gene-a (GROa) or epithelial cell dermid neutrophil-activating protein-78 (ENA-78).

Reverse transcription polymerase chain reaction (RT-PCR) analysis of $\alpha E N a C$ mRNA expression. A total of $100 \mathrm{mg}$ fresh lung tissue homogenate was prepared using TRIzol ${ }^{\circledR}$ reagent (Invitrogen Life Technologies, Grand Island, NY, USA) according to the manufacturer's instructions. Purified RNA was extracted and RT-PCR was performed using primer sequences targeting $\alpha \mathrm{ENaC}$. The primer sequences were as follows: 5'-CCATGAAGGGCAACCAAT-3' (forward) and 5'-CGAACAGCAAGGCGAACT-3' (reverse). $\beta$-actin was used as an internal control with the primer sequences: 5'-AGCGGGAAATCGTGCGTGACATT-3' (forward) and 5'-CAGGAAGGAAGGCTGGAAGAGTG-3' (reverse). Amplification was performed using the following conditions: $50^{\circ} \mathrm{C}$ for $30 \mathrm{~min}, 95^{\circ} \mathrm{C}$ for $5 \mathrm{~min}, 95^{\circ} \mathrm{C}$ denaturation for $30 \mathrm{sec}$, $60^{\circ} \mathrm{C}$ annealing for $30 \mathrm{sec}$ and $72^{\circ} \mathrm{C}$ extension for $1 \mathrm{~min}$, for 20 and 25 cycles. RT-PCR products were separated using agarose gel electrophoresis. A gel imaging analysis system was used to determine the integrated optical density values of $\alpha \mathrm{ENaC}$ and $\beta$-actin and the quantity of $\alpha$-ENaC mRNA was determined relative to the integrated optical density value of $\beta$-actin mRNA.

Western blot analysis of $\alpha E N a C$ protein expression. Protein lysates were prepared according to standard protocols. Lung tissues were washed thoroughly using PBS, prior to homogenization at a dilution of 1:10 (v/v) in a lysis buffer containing $50 \mathrm{mM}$ Tris (pH 7.4), $150 \mathrm{mM} \mathrm{NaCl}, 0.5 \%$ NP-40, $50 \mathrm{mM} \mathrm{NaF}, 1 \mathrm{mM} \mathrm{Na} \mathrm{VO}_{4}, 1 \mathrm{mM}$ phenylmethylsul- fonyl fluoride, $25 \mathrm{mg} / \mathrm{ml}$ leupeptin and $25 \mathrm{mg} / \mathrm{ml}$ aprotinin. Lysates were then centrifuged at 10,000 x g and the supernatants were collected. Equal quantities of protein lysates were used for western blot analyses with the indicated antibodies. Protein lysates (30 $\mu \mathrm{g}$ protein/lane) were boiled for $5 \mathrm{~min}$ at $95^{\circ} \mathrm{C}$, prior to being separated on an $8 \%$ SDS-polyacrylamide gel and transferred to a nitrocellulose membrane. Membranes were blocked using 5\% milk in tris-buffered saline (TBS) for $2 \mathrm{~h}$ and subsequently washed with $0.05 \%$ Tween-20-TBS and incubated overnight at $4{ }^{\circ} \mathrm{C}$ with a 1:200 dilution of anti- $\alpha \mathrm{ENaC}$ antibody (Santa Cruz Biotechnology, Inc., Santa Cruz, CA, USA). Membranes were then washed and incubated with peroxidase-conjugated affinity-purified goat anti-mouse immunoglobulin $\mathrm{G}$ ( $\mathrm{IgG}$ ) and goat anti-rabbit IgG secondary antibodies at a 1:20,000 dilution (Jackson ImmunoResearch Laboratories, Inc., West Grove, MA, USA) for $2 \mathrm{~h}$ at $37^{\circ} \mathrm{C}$. Protein bands were visualized using an enhanced chemiluminescence (ECL) system (GE Healthcare, Waukesha, WI, USA) and quantified by densitometry. $\beta$-actin (Santa Cruz Biotechnology, Inc.) was used as a loading control at a 1:3,000 dilution. All experiments were repeated at least three times.

Statistical analyses. Results are presented as the mean \pm standard deviation. Results for normally distributed continuous variables were compared using one-way analysis of variance (ANOVA). When a significant difference between groups was detected, multiple mean comparisons were performed using the Bonferroni procedure with a type-I error adjustment. All statistical analyses were two-sided and performed using SPSS 15.0 statistics software (SPSS, Inc., Chicago, IL, USA). $\mathrm{P}<0.05$ was considered to indicate a statistically significant difference.

\section{Results}

Lung histology. Histopathological examination of HE-stained lung tissue sections from rats in the sham, sham + PNS, ALI and ALI + PNS groups are shown in Fig. 1A-D, respectively. ALI rats were observed to demonstrate the characteristic features of pulmonary injury, whereas rats in the sham and sham + PNS groups demonstrated normal alveolar architecture. Specifically, rats in the ALI group exhibited an increased thickness of the alveolar wall, pulmonary edema and hemorrhage, as well as infiltration of inflammatory cells into the alveolar spaces (Fig. 1C). PNS treatment in ALI rats was observed to prevent pulmonary parenchymal damage and the lung histology in these rats was identified to be similar to that of the sham control rats, with numerous distended alveoli with thin, flattened, delicate walls. Furthermore, in the rats in the ALI + PNS group, alveoli were observed to be well aerated and with few neutrophils within the interstitium (Fig. 1D).

BALF collection and cell counts. As shown in Fig. 2, at $2 \mathrm{~h}$ subsequent to treatment, no significant differences were observed in the total leukocyte count or the percentage of polymorphonuclear neutrophils (PMN) in the BALF between the sham and sham + PNS groups (both $\mathrm{P}=1.000$ ). However, the ALI group, in which OA-LPS injury had been induced, exhibited significantly higher total leukocyte counts and 
A

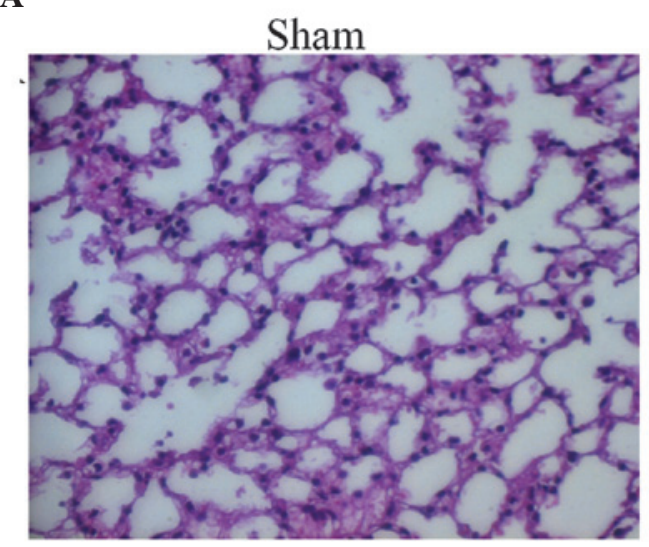

C

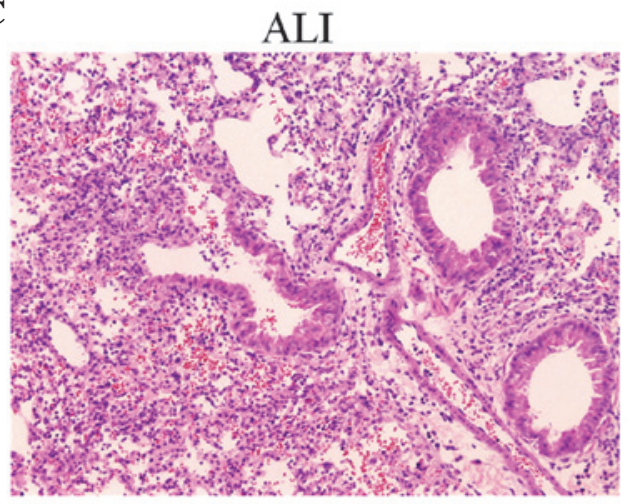

B

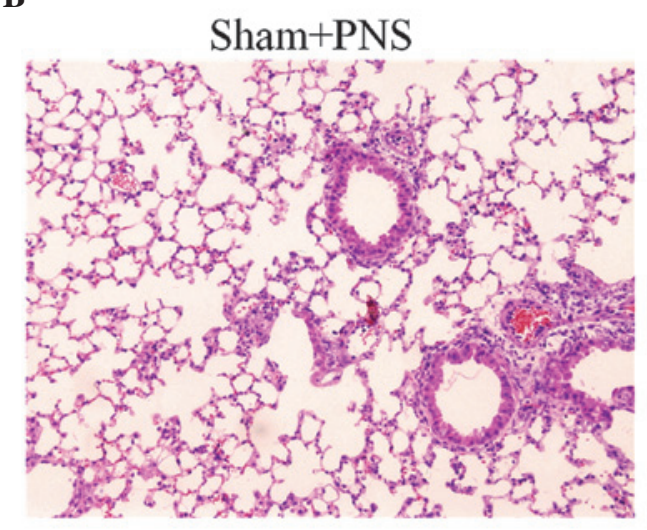

D

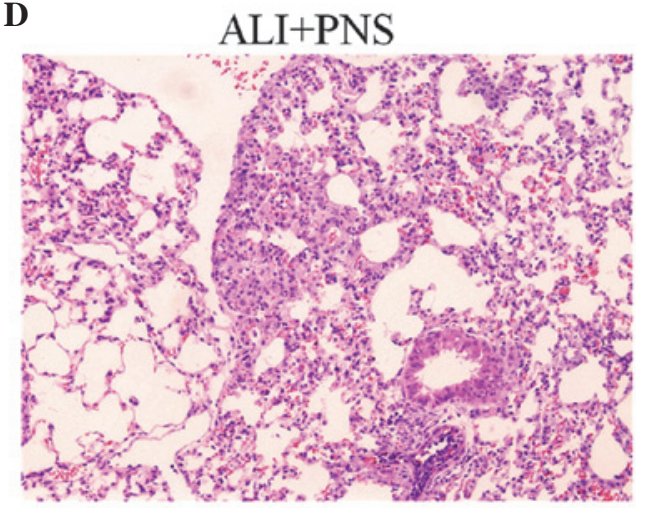

Figure 1. Histopathological examinations of H\&E-stained lung tissue sections. Lung histology of rats subjected to sham injection with or without treatment with PNS and injection of OA followed by $4 \mathrm{~h}$ of LPS injection, with or without treatment with PNS. (A) Sham group (magnification, x100). (B) Sham with PNS treatment group (magnification, $x 100$ ). (C) OA-LPS-induced ALI group (magnification, $x 400$ ). Thickened interstitial walls and a dense inflammatory cell infiltrate were observed. (D) ALI with PNS treatment group (magnification, x400). Relatively normal histology with thin alveolar walls and few infiltrating inflammatory cells. PNS, Panax notoginseng saponins; ALI, acute lung injury; H\&E, hematoxylin and eosin; LPS, lipopolysaccharide; OA, oleic acid.

PMN percentages in the BALF, compared with those of the sham groups (all $\mathrm{P}<0.001)$. Following PNS administration (ALI + PNS group), ALI rats demonstrated significantly reduced total leukocyte counts and PMN percentages in the BALF compared with the ALI group (both $\mathrm{P}<0.001$ ).

Partial pressure $(\mathrm{Pa}) \mathrm{O}_{2} / \mathrm{FiO}_{2}$ ratio and $\mathrm{PaCO}$. As shown in Fig. 3 no significant difference was observed in the $\mathrm{PaO}_{2} / \mathrm{FiO}_{2}$ ratio in the sham + PNS group compared with the sham group. By contrast, rats in the ALI and ALI + PNS groups exhibited significantly lower $\mathrm{PaO}_{2} / \mathrm{FiO}_{2}$ ratios ( $\mathrm{P}<0.001$; Fig. $\left.3 \mathrm{~A}\right)$. However, PNS administration was not observed to significantly improve the $\mathrm{PaO}_{2} / \mathrm{FiO}_{2}$ ratio in ALI rats $(\mathrm{P}=0.172)$. No significant differences were observed in the $\mathrm{PaCO}_{2}$ values among the rats in the four groups $(\mathrm{P}=0.865$; Fig. $3 \mathrm{~B})$

Lung W/D weight ratio and EVLW. A significantly increased lung W/D weight ratio was observed in the rats in the ALI and ALI + PNS groups compared with those in the sham and sham + PNS groups $(\mathrm{P}<0.001$; Fig. 4A). Moreover, the EVLW level was identified to be significantly higher in the rats in the ALI and ALI + PNS groups compared with those in the sham and sham + PNS groups $(\mathrm{P}<0.001$; Fig. 4B). However, following administration of PNS in ALI rats, the EVLW level in lung tissues was observed to significantly decrease compared with the ALI rats that had not received PNS treatment $(\mathrm{P}=0.026$; Fig. 4B).

Serum and BALF cytokine levels. Serum levels of TNF- $\alpha$, IL-6 and IL-10 (Fig. 5A-C, respectively) were observed to be significantly increased in rats in the ALI and ALI + PNS groups compared with those in the sham and sham + PNS groups (all $\mathrm{P}<0.001$ ). However, no significant differences were detected in the levels of TNF- $\alpha$, IL- 6 and IL-10 in the sera of rats between the sham and the sham + PNS group. A similar pattern of cytokine concentration was observed in the BALF of the four groups of rats. However, compared with the ALI group, the levels of TNF- $\alpha$, IL-6 and IL-10 were significantly decreased in the sera and BALF of ALI rats following PNS administration (all $\mathrm{P}<0.001$ ).

$\alpha E N a C m R N A$ and protein expression. As shown in Fig. 6, no significant differences were observed in the levels of $\alpha \mathrm{ENaC}$ mRNA (Fig. 6A) and protein (Fig. 6B) expression in left lung tissues between the rats in the sham and the sham + PNS groups. However, it was observed that OA-LPS-induced ALI significantly decreased the mRNA and protein expression of $\alpha \mathrm{ENaC}$ in rat left lung tissues. Moreover, following PNS administration, a significant increase in $\alpha E N a C$ mRNA and protein expression was observed in the left lung tissue 
A

Total cell number

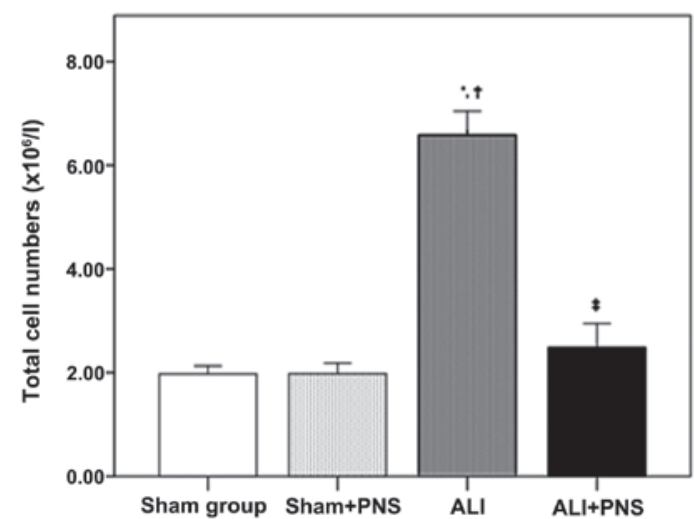

B Polymorphonuclear neutrophils number

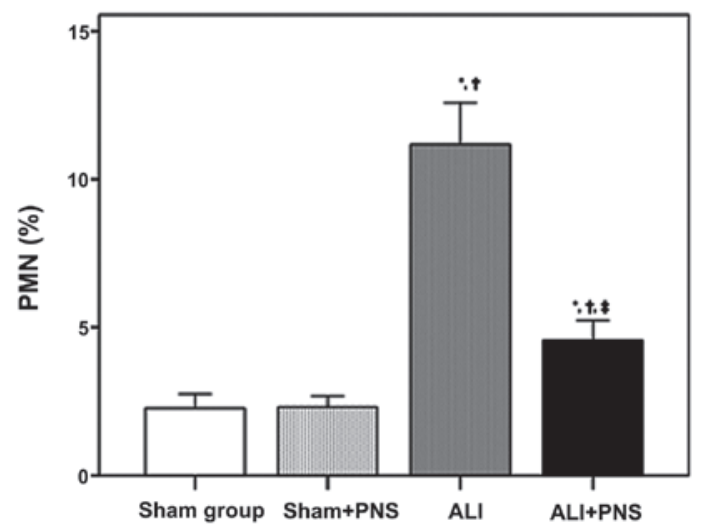

Figure 2. PNS treatment reduces total leukocytes and PMNs in the BALF, following OA-LPS-induced ALI. BALF samples were obtained from four groups of seven rats: sham; sham+PNS; ALI and ALI+PNS. BALF samples were examined for (A) total leukocyte counts and (B) percentages of PMNs among leukocytes. " $\mathrm{P}<0.05$ between the indicated group and the sham group. ${ }^{\circ} \mathrm{P}<0.05$ between the indicated group and the sham+PNS group. ${ }^{*} \mathrm{P}<0.05$ between the ALI and ALI+PNS groups. Pair-wise multiple comparisons were performed between groups using Bonferroni's test with $\alpha=0.017$ adjustment. PNS, Panax notoginseng saponins; ALI, acute lung injury; LPS, lipopolysaccharide; OA, oleic acid; PMN, polymorphonuclear neutrophils; BALF, bronchoalveolar lavage fluid.
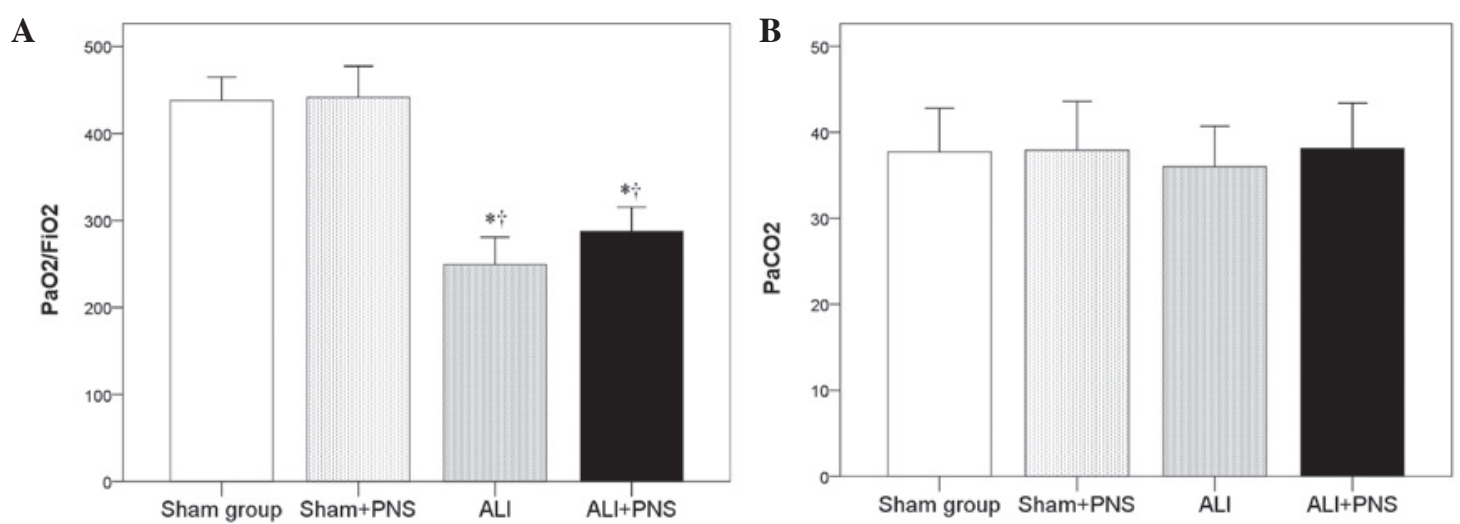

Figure 3. PNS treatment improves lung oxygenation following OA-LPS-induced ALI. Arterial blood samples were obtained from four groups of seven rats: sham; sham+PNS; ALI and ALI+PNS. Samples were assayed for (A) $\mathrm{PaO}_{2} / \mathrm{FiO}_{2}$ and (B) $\mathrm{PaCO}_{2}$ levels. ${ }^{*} \mathrm{P}<0.05$ between the indicated group and the sham group. ${ }^{\dagger} \mathrm{P}<0.05$ between the indicated group and the sham+PNS group. ${ }^{\dagger} \mathrm{P}<0.05$ between the ALI and ALI+PNS groups. Pair-wise multiple comparisons were performed between groups using Bonferroni's test with $\alpha=0.017$ adjustment. PNS, Panax notoginseng saponins; ALI, acute lung injury; LPS, lipopolysaccharide; $\mathrm{OA}$, oleic acid; $\mathrm{Pa}$, partial pressure; $\mathrm{FiO}_{2}$, fraction of inspired oxygen.
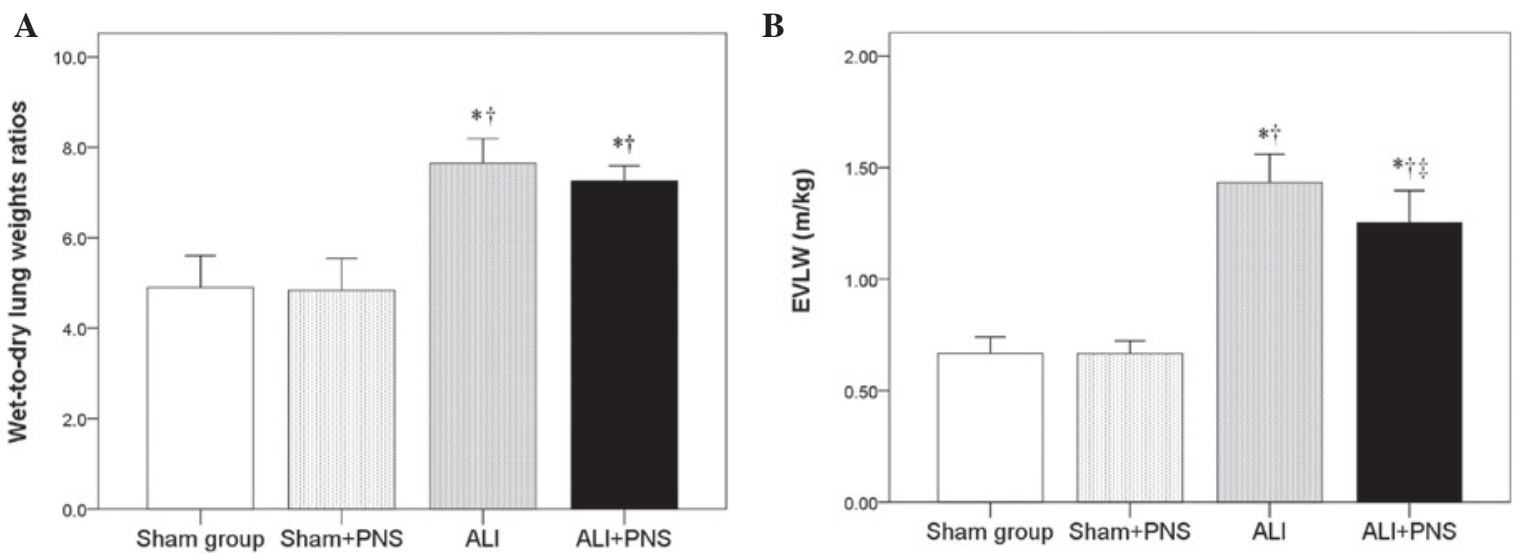

Figure 4. Effect of PNS on lung W/D weight ratios and EVLW following OA-LPS-induced ALI. Right lungs were harvested from four groups of seven rats: sham; sham+PNS; ALI and ALI+PNS. (A) W/D lung weight ratios and (B) EVLW were measured. "P $<0.05$ between the indicated group and the sham group. ${ }^{\top} \mathrm{P}<0.05$ between the indicated group and the sham+PNS group. ${ }^{*} \mathrm{P}<0.05$ between the ALI and ALI+PNS groups. Pair-wise multiple comparisons were performed between groups using Bonferroni's test with $\alpha=0.017$ adjustment. PNS, Panax notoginseng saponins; ALI, acute lung injury; LPS, lipopolysaccharide; OA, oleic acid; W/D, wet-to-dry; EVLW, extravascular lung water. 


\section{A TNFa}

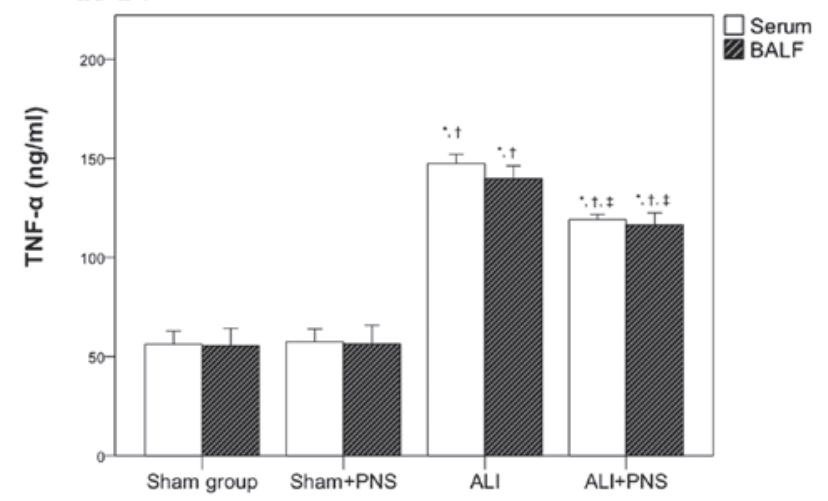

B IL-6

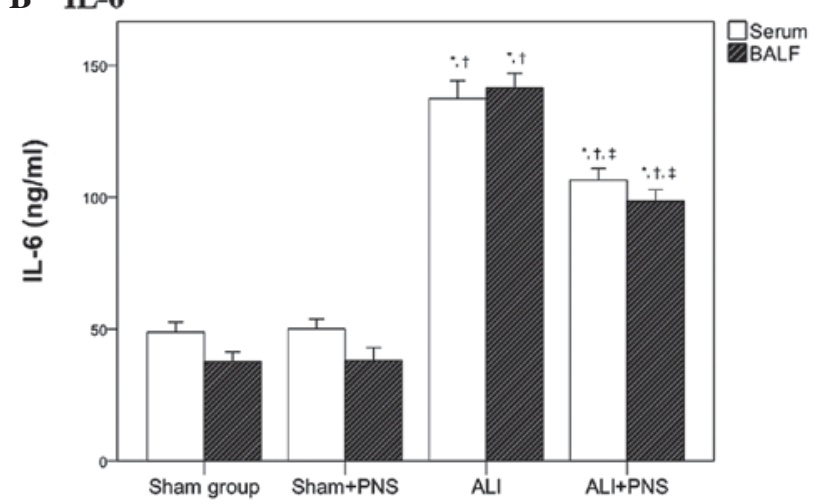

C $\quad$ L-10

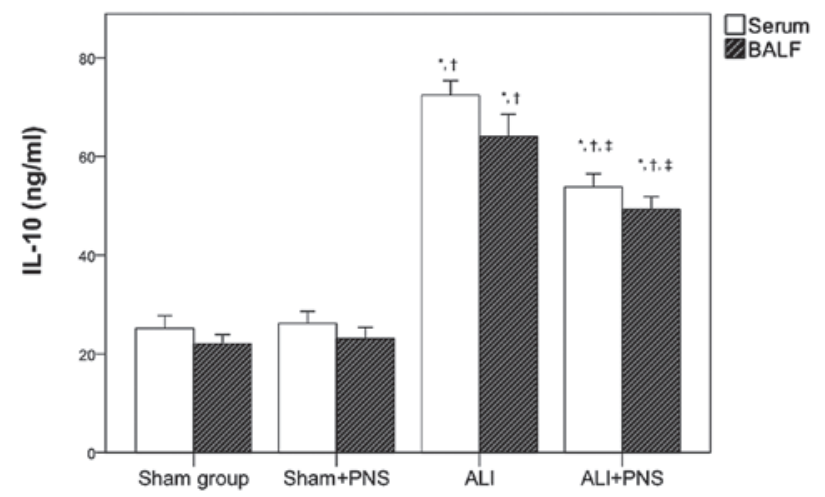

Figure 5. Effect of PNS treatment on levels of inflammatory cytokines in the serum and BALF of OA-LPS-induced ALI rats. Serum and BALF samples were obtained from four groups of seven rats: sham; sham+PNS; ALI and ALI+PNS. ELISAs were performed to determine the concentrations of (A) TNF- $\alpha$, (B) IL-6 and (C) IL-10. " $\mathrm{P}<0.05$ between the indicated group and the sham group. ${ }^{ } \mathrm{P}<0.05$ between the indicated group and the sham+PNS group. ${ }^{*} \mathrm{P}<0.05$ between the ALI and ALI+PNS groups. Pair-wise multiple comparisons were performed between groups using Bonferroni's test with $\alpha=0.017$ adjustment. PNS, Panax notoginseng saponins; ALI, acute lung injury; LPS, lipopolysaccharide; OA, oleic acid; BALF, bronchoalveolar lavage fluid; TNF- $\alpha$, tumor necrosis factor- $\alpha$; IL, interleukin.

of ALI rats compared with ALI rats without PNS treatment $(\mathrm{P}=0.017)$.

\section{Discussion}

This study has demonstrated that OA injection followed by LPS administration is capable of inducing severe pulmonary parenchymal damage in rats, consistent with various adverse effects on lung water homeostasis that are associated with ALI in animal models $(8,23)$. OA has been reported to inhibit alveolar fluid reabsorption (24) and the endotoxin, LPS, has been indicated to adversely affect $\mathrm{ENaC}$-mediated $\mathrm{Na}^{+}$transport $(25,26)$.

In the present study, PNS treatment was observed to ameliorate some of the ALI-induced effects within a clinically relevant time frame. PNS treatment was observed to decrease the lung EVLW level in ALI rats and partially restore the reduced $\alpha \mathrm{ENaC}$ mRNA and protein levels detected in ALI lung tissue. Thus, PNS treatment may ameliorate some of the adverse characteristics associated with lung water homeostasis in ALI through improving AFC, which is a function of transepithelial $\mathrm{Na}^{+}$transport (5). Furthermore, the results of this study identified that PNS may attenuate the inflammation associated with OA-LPS injury in rats.

Neutrophil infiltration has been reported to have a significant role in the development of pulmonary edema and microvascular leakage in animal models. Moreover, activated neutrophils are suggested to be the primary mediators of local and remote tissue damage induced by II/R $(29,30)$. The results of this study show that PNS treatment is capable of significantly reducing the total leukocyte and PMN counts in the lungs of ALI rats (Fig. 2).

ALI and its more severe form, ARDS, are characterized by an excessive inflammatory response in the lung, which is associated with, and may be promoted by, an increase in inflammatory cytokines in the plasma $(5,31)$. Previous clinical investigations have revealed a positive correlation between mortality and levels of circulating TNF- $\alpha$, IL-1 $\beta$, IL- 6 and IL-8 in ALI (22-35). In the present study, the reduction in inflammatory cytokine levels observed in the plasma of ALI rats following PNS treatment may have contributed to a reduction in leukocyte adhesion and infiltration. In ALI rats, PNS treatment was observed to reduce neutrophil infiltration into the lungs; however, infiltration levels were still greater than those observed in the sham and sham + PNS groups of rats.

In a study by Sun et al (36), PNS treatment was reported to reduce LPS-induced leukocyte adhesion in rat mesenteric venules by inhibiting the expression of the adhesion molecules cluster of differentiation molecule 11B (CD11b) and integrin $\beta-2$ (CD18) in neutrophils. In a previous study, we showed that PNS administration decreased bacterial translocation and reduced pulmonary parenchymal damage, following II/R in rats. Furthermore, PNS was observed to reduce the activity of oxidative enzymes, the levels of MDA and nitric oxide, the activity of inducible nitric oxide synthase in the lung tissue and the levels of pro-inflammatory cytokines in the plasma (22).

In addition to its effects on lung water homeostasis, the OA-LPS model may also induce severe ALI through its effects on lung inflammatory responses (23). These effects may be associated with abnormally increased levels of pro-inflammatory cytokines. Experimental studies suggest that cytokine responses are normally compartmentalized within the lungs; therefore, the study of blood samples may provide an incomplete representation of the inflammatory events within the lungs (37). However, it has been suggested that compartmentalization is lost to some extent during severe inflammatory responses (38), although measuring cytokine levels within the lungs is likely to be more accurate than measuring those in plasma or serum samples. Cytokines are active in the alveolar compartment, where they may exist as soluble constituents of 
A

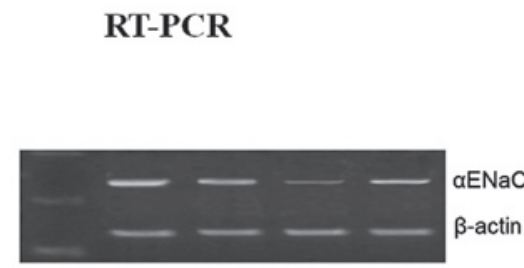

B
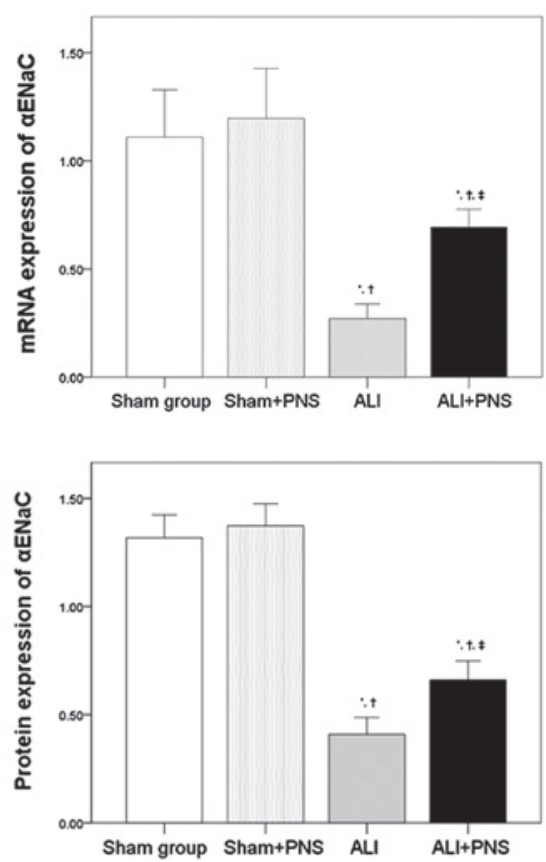

Figure 6. PNS treatment partially restores $\alpha \mathrm{ENaC}$ mRNA and protein expression in lung tissue following OA-LPS-induced ALI. Left lung tissues were harvested from four groups of seven rats: sham; sham+PNS; ALI and ALI+PNS. (A) $\alpha E N a C$ mRNA and (B) $\alpha E N a C$ protein expression was determined using RT-PCR and western blot analysis, respectively. ${ }^{*} \mathrm{P}<0.05$ between the indicated group and the sham group. ${ }^{~} \mathrm{P}<0.05$ between the indicated group and the sham+PNS group. ${ }^{\circ} \mathrm{P}<0.05$ between the ALI and ALI+PNS groups. Pair-wise multiple comparisons were performed between groups using Bonferroni's test with $\alpha=0.017$ adjustment. PNS, Panax notoginseng saponins; ALI, acute lung injury; LPS, lipopolysaccharide; OA, oleic acid; ENaC, epithelial sodium channel; RT-PCR, reverse transcription polymerase chain reaction.

alveolar fluids, as well as in the tissue compartment; therefore, sampling cytokines in the lungs may be problematic. Sampling alveolar fluids using BAL may represent an alternative technique for measuring the concentration and function of specific cytokines in the lungs (39).

Of note, TNF- $\alpha$ has been identified to stimulate cytokine production by lung epithelial and mesenchymal cells that are unresponsive to bacteria and their products directly (40). Suter et al (41) found significant levels of TNF- $\alpha$ in the lung fluid of patients at the onset of ARDS. TNF- $\alpha$ mediates its effects through two TNF- $\alpha$ receptors: TNF-receptor I (RI) and TNF-RII. Park et al (16) demonstrated that the concentrations of TNFR-I and TNFR-II exceeded the concentration of immunoreactive TNF- $\alpha$ in BALF during the course of ARDS. Furthermore, they observed that the activity of TNF- $\alpha$ was effectively inhibited in the aqueous phase of lung fluids of patients with ARDS. TNF- $\alpha$ has also been suggested to induce the release of IL- 6 by endothelial cells and macrophages (42).

IL-6 was originally identified as a B-cell growth factor (43). IL- 6 is produced by activated macrophages and stimulates acute-phase responses in the liver. IL- 6 production is induced, in part, by TNF- $\alpha$ and IL- $1 \beta$ and it has been proposed that IL- 6 integrates signals produced in the early stages of the inflammatory response (16). Experimental studies have identified that, in the BALF of patients at risk for ARDS, IL-6 concentrations are high and remain elevated throughout the course of established ARDS. It has been reported that the IL- 6 receptor, IL-6R, is released from cell membranes and enters the circulation. Martin (14) observed that the concentration of soluble IL-6R was elevated in the BALF of patients at risk of ARDS and throughout the course of the condition. Therefore, cellular reactions mediated by IL- 6 may be of significance during ARDS (14).

In the present study the levels of TNF- $\alpha$ and IL- 6 were observed to be significantly higher in the BALF of rats in the ALI groups compared with those in the sham groups $(\mathrm{P}<0.05$; Fig. 5). Of note, TNF- $\alpha$ and IL-6 levels were found to be significantly lower in the BALF and serum of ALI rats following PNS administration, which may have contributed in alleviating subsequent systemic inflammatory responses.

IL-10 is a counter-regulatory cytokine that inhibits cytokine production by stimulated macrophages $(44,45)$. IL-10 is detectable in ARDS BALF and it has been reported that IL-10 treatment is capable of life extension in animals $(46,47)$. Donnelly et al (48) found that patients who died with ARDS had low concentrations of IL-10 in the BALF at the onset of ARDS, suggesting inadequate attenuation of lung inflammatory responses. Lo et al (49) observed that IL-10 inhibited the production of TNF by LPS-stimulated macrophages. Furthermore, IL-10 has been reported to reduce TNF mRNA expression (49). In the present study, PNS treatment was also observed to decrease IL-10 levels in the BALF and serum, in addition to those of TNF- $\alpha$ and IL-6. Therefore, PNS administration may decrease the subsequent systemic inflammatory response and may aid in preventing ALI from developing to ARDS. To summarize, following OA-LPS treatment, the levels of IL-10 in the serum and BALF of ALI rats were markedly higher than those in rats in the PNS-treated group. These findings suggest that ALI may be associated with pro- and anti-inflammatory responses and that an imbalance between these responses may be a major mechanism 
underlying the deterioration associated with ALI and the progression to ARDS. The anti-inflammatory effects of PNS are well established, and in the present study, PNS was not only found to reduce TNF- $\alpha$ and IL- 6 levels in the lungs of rats with OA-LPS-induced ALI, but also significantly decrease IL-10 levels in the lungs. These findings suggest that PNS may not only improve lung inflammation, but also aid in balancing pro- and anti-inflammatory responses, which may limit progression from ALI to ARDS.

It has been shown that PNS may exert protective effects against cerebral ischemia, be beneficial for the cardiovascular system and possess hemostatic, antioxidant and estrogen-like activities (19-21). We previously reported that PNS treatment (100 mg/kg) was capable of alleviating II/R-induced ALI in rats and significantly reduced the activity of oxidant enzymes and inducible nitric oxide synthase in the lung, as well as the levels of MDA and nitric oxide, and pro-inflammatory cytokines in the plasma (22).

It has previously been demonstrated that extracts from certain naturally derived substances may be capable of attenuating diseases associated with adverse inflammatory responses, including models of ALI. Chen et al (29) reported that pre-treatment with $30 \mathrm{mg} / \mathrm{kg}$ Radix Paeoniae Rubra (RPR), injected into the femoral vein for $2 \mathrm{~h}, 4 \mathrm{~h}$ prior to reperfusion, was capable of attenuating ALI induced by $1 \mathrm{~h}$ of intestinal ischemia followed by $2 \mathrm{~h}$ of reperfusion in rats. The RPR treatment was observed to decrease MDA levels and prevent the decrease in superoxide dismutase (SOD) activity in the lungs (29). Furthermore, a study by Peng et al (50) reported that PNS treatment had anti-fibrotic effects in a rat model of hepatic fibrosis as a consequence of altered expression of the same cytokines assessed in the present study, namely TNF- $\alpha$, IL-6 and IL-10. In accordance with this finding, Lee et al (51) found that Korean red ginseng ameliorated focal cerebral ischemia/reperfusion injury in rats and that these neuroprotective effects were a result of altered inflammatory cytokine levels. The present study showed that in a model of ALI, PNS treatment demonstrated anti-inflammatory properties with a similar cytokine profile to that reported previously, and prevented the reduction in $\alpha \mathrm{ENaC}$ mRNA and protein levels in the lung tissue. As this was an exploratory investigation, only the $\alpha$ subunit of $\mathrm{ENaC}$ was investigated; however, of note, it has been reported that the $\beta$ and $\gamma$ subunits may be involved in rat models of ALI (8). Further in-depth investigations are required to elucidate the complex interrelation between cytokines, $\mathrm{ENaCs}$ and PNS in the damage associated with ALI.

In conclusion, this study showed that PNS administration was capable of ameliorating OA-LPS-induced ALI in Wistar rats. These effects were primarily a consequence of the anti-inflammatory properties of PNS. The capacity of PNS to alleviate ALI if administered following OA-LPS injection in this model has yet to be investigated. The present study has highlighted the requirement for further investigation into the protective effects of PNS, as well as its clinical use in OA-LPS-induced ALI. This may lead to the development of PNS as a therapeutic agent for ALI and, potentially, its more severe manifestation, ARDS.

\section{Acknowledgements}

This study was supported by the National Natural Science Foundation of China (no. 81170028).

\section{References}

1. Bernard GR, Artigas A, Brigham KL, et al: The American-European Consensus Conference on ARDS. Definitions, mechanisms, relevant outcomes, and clinical trial coordination. Am J Respir Crit Care Med 149: 818-824, 1994.

2. Fowler AA, Hamman RF, Good JT, et al: Adult respiratory distress syndrome: risk with common predispositions. Ann Intern Med 98: 593-597, 1983.

3. Pepe PE, Potkin RT, Reus DH, Hudson LD and Carrico CJ: Clinical predictors of the adult respiratory distress syndrome. Am J Surg 144: 124-130, 1982.

4. Rinaldo JE and Christman JW: Mechanisms and mediators of the adult respiratory distress syndrome. Clin Chest Med 11: 621-632, 1990.

5. Ware LB and Matthay MA: Alveolar fluid clearance is impaired in the majority of patients with acute lung injury and the acute respiratory distress syndrome. Am J Respir Crit Care Med 163: 1376-1383, 2001

6. Matthay MA, Robriquet L and Fang X: Alveolar epithelium: role in lung fluid balance and acute lung injury. Proc Am Thorac Soc 2: 206-213, 2005.

7. Canessa CM, Schild L, Buell G, et al: Amiloride-sensitive epithelial $\mathrm{Na}^{+}$channel is made of three homologous subunits. Nature 367: 463-467, 1994.

8. Deng W, Li CY, Tong J, Zhang W and Wang DX: Regulation of ENaC-mediated alveolar fluid clearance by insulin via PI3K/Akt pathway in LPS-induced acute lung injury. Respir Res 13: 29, 2012.

9. Bellingan GJ: The pulmonary physician in critical care * 6: The pathogenesis of ALI/ARDS. Thorax 57: 540-546, 2002.

10. Goodman RB, Strieter RM, Martin DP, et al: Inflammatory cytokines in patients with persistence of the acute respiratory distress syndrome. Am J Respir Crit Care Med 154: 602-611, 1996.

11. Tomashefski JF Jr: Pulmonary pathology of acute respiratory distress syndrome. Clin Chest Med 21: 435-466, 2000.

12. Johnson JL, Moore EE, Tamura DY, Zallen G, Biffl WL and Silliman CC: Interleukin-6 augments neutrophil cytotoxic potential via selective enhancement of elastase release. J Surg Res 76: 91-94, 1998.

13. Kiehl MG, Ostermann H, Thomas M, Müller C, Cassens U and Kienast J: Inflammatory mediators in bronchoalveolar lavage fluid and plasma in leukocytopenic patients with septic shock-induced acute respiratory distress syndrome. Crit Care Med 26: 1194-1199, 1998.

14. Martin TR: Lung cytokines and ARDS: Roger S. Mitchell Lecture. Chest 116 (Suppl 1): 2S-8S, 1999.

15. Meduri GU, Kanangat S, Stefan J, Tolley E and Schaberg D: Cytokines IL-1beta, IL-6, and TNF-alpha enhance in vitro growth of bacteria. Am J Respir Crit Care Med 160: 961-967, 1999.

16. Park WY, Goodman RB, Steinberg KP, et al: Cytokine balance in the lungs of patients with acute respiratory distress syndrome. Am J Respir Crit Care Med 164: 1896-1903, 2001.

17. Headley AS, Tolley E and Meduri GU: Infections and the inflammatory response in acute respiratory distress syndrome. Chest 111: 1306-1321, 1997.

18. Meduri GU, Kohler G, Headley S, Tolley E, Stentz F and Postlethwaite A: Inflammatory cytokines in the BAL of patients with ARDS. Persistent elevation over time predicts poor outcome. Chest 108: 1303-1314, 1995.

19. Li SH and Chu Y: Anti-inflammatory effects of total saponins of Panax notoginseng. Zhongguo Yao Li Xue Bao 20: 551-554, 1999.

20. Ng TB: Pharmacological activity of sanchi ginseng (Panax notoginseng). J Pharm Pharmacol 58: 1007-1019, 2006.

21. Sun K, Wang CS, Guo J, et al: Protective effects of ginsenoside $\mathrm{Rb} 1$, ginsenoside $\mathrm{Rg} 1$, and notoginsenoside R1 on lipopolysaccharide-induced microcirculatory disturbance in rat mesentery. Life Sci 81: 509-518, 2007.

22. Rong L, Chen Y, He M and Zhou X: Panax notoginseng saponins attenuate acute lung injury induced by intestinal ischaemia/reperfusion in rats. Respirology 14: 890-898, 2009.

23. Wang HM, Bodenstein M and Markstaller K: Overview of the pathology of three widely used animal models of acute lung injury. Eur Surg Res 40: 305-316, 2008.

24. Vadász I, Morty RE, Kohstall MG, et al: Oleic acid inhibits alveolar fluid reabsorption: a role in acute respiratory distress syndrome? Am J Respir Crit Care Med 171: 469-479, 2005. 
25. Adebamiro A, Cheng Y, Johnson JP and Bridges RJ: Endogenous protease activation of $\mathrm{ENaC}$ : effect of serine protease inhibition on ENaC single channel properties. J Gen Physiol 126: 339-352, 2005.

26. Baines DL, Albert AP, Hazell MJ, Gambling L, Woollhead AM and Dockrell ME: Lipopolysaccharide modifies amiloride-sensitive $\mathrm{Na}^{+}$transport processes across human airway cells: role of mitogen-activated protein kinases ERK 1/2 and 5. Pflugers Arch 459: 451-463, 2010.

27. Shen JF, Qiu HB, Yang Y, et al: Comparison of single-indicator thermodilution versus gravimetric measurement in determination of extra-vascular lung water in dogs with acute respiratory distress syndrome. Zhongguo Wei Zhong Bing Ji Jiu Yi Xue 18: 327-330, 2006 (In Chinese).

28. Walley KR, Lukacs NW, Standiford TJ, Strieter RM and Kunkel SL: Balance of inflammatory cytokines related to severity and mortality of murine sepsis. Infect Immun 64: 4733-4738, 1996.

29. Chen C, Zhang F, Xia ZY, Lin H and Mo AS: Protective effects of pretreatment with Radix Paeoniae Rubra on acute lung injury induced by intestinal ischemia/reperfusion in rats Chin J Traumatol 11: 37-41, 2008.

30. Türüt H, Kurutas EB, Bulbuloglu E, et al: Zinc aspartate alleviates lung injury induced by intestinal ischemia-reperfusion in rats. J Surg Res 151: 62-67, 2009.

31. Ware LB and Matthay MA: The acute respiratory distress syndrome. N Engl J Med 342: 1334-1349, 2000.

32. Casey LC, Balk RA and Bone RC: Plasma cytokine and endotoxin levels correlate with survival in patients with the sepsis syndrome. Ann Intern Med 119: 771-778, 1993.

33. Hack CE, Hart M, van Schijndel RJ, et al: Interleukin-8 in sepsis: relation to shock and inflammatory mediators. Infect Immun 60 2835-2842, 1992.

34. Meduri GU, Headley S, Kohler G, et al: Persistent elevation of inflammatory cytokines predicts a poor outcome in ARDS Plasma IL-1 beta and IL-6 levels are consistent and efficient predictors of outcome over time. Chest 107: 1062-1073, 1995.

35. Pinsky MR, Vincent JL, Deviere J, Alegre M, Kahn RJ and Dupont E: Serum cytokine levels in human septic shock. Relation to multiple-system organ failure and mortality. Chest 103 : $565-575,1993$

36. Sun K, Wang CS, Guo J, et al: Effect of Panax notoginseng saponins on lipopolysaccharide-induced adhesion of leukocytes in rat mesenteric venules. Clin Hemorheol Microcirc 34: 103-108, 2006.

37. Nelson S, Bagby GJ, Bainton BG, Wilson LA, Thompson JJ and Summer WR: Compartmentalization of intraalveolar and systemic lipopolysaccharide-induced tumor necrosis factor and the pulmonary inflammatory response. J Infect Dis 159: 189-194, 1989.

38. Tutor JD, Mason CM, Dobard E, Beckerman RC, Summer WR and Nelson S: Loss of compartmentalization of alveolar tumor necrosis factor after lung injury. Am J Respir Crit Care Med 149: $1107-1111,1994$
39. Steinberg KP, Mitchell DR, Maunder RJ, Milberg JA, Whitcomb ME and Hudson LD: Safety of bronchoalveolar lavage in patients with adult respiratory distress syndrome. Am Rev Respir Dis 148: 556-561, 1993.

40. Standiford TJ, Kunkel SL, Phan SH, Rollins BJ and Strieter RM: Alveolar macrophage-derived cytokines induce monocyte chemoattractant protein-1 expression from human pulmonary type II-like epithelial cells. J Biol Chem 266: 9912-9918, 1991.

41. Suter PM, Suter S, Girardin E, Roux-Lombard P, Grau GE and Dayer JM: High bronchoalveolar levels of tumor necrosis factor and its inhibitors, interleukin-1, interferon, and elastase, in patients with adult respiratory distress syndrome after trauma, shock, or sepsis. Am Rev Respir Dis 145: 1016-1022, 1992.

42. Moriceau G, Ory B, Gobin B, et al: Therapeutic approach of primary bone tumours by bisphosphonates. Curr Pharm Des 16: 2981-2987, 2010.

43. Papanicolaou DA, Wilder RL, Manolagas SC and Chrousos GP The pathophysiologic roles of interleukin-6 in human disease. Ann Intern Med 128: 127-137, 1998.

44. Fiorentino DF, Zlotnik A, Mosmann TR, Howard M and O'Garra A: IL-10 inhibits cytokine production by activated macrophages. J Immunol 147: 3815-3822, 1991.

45. Ramani M, Ollivier V, Khechai F, et al: Interleukin-10 inhibits endotoxin-induced tissue factor mRNA production by human monocytes. FEBS Lett 334: 114-116, 1993.

46. Standiford TJ, Strieter RM, Lukacs NW and Kunkel SL: Neutralization of IL-10 increases lethality in endotoxemia. Cooperative effects of macrophage inflammatory protein-2 and tumor necrosis factor. J Immunol 155: 2222-2229, 1995.

47. van der Poll T, Marchant A, Buurman WA, et al: Endogenous IL-10 protects mice from death during septic peritonitis. J Immunol 155: 5397-5401, 1995.

48. Donnelly SC, Strieter RM, Reid PT, et al: The association between mortality rates and decreased concentrations of interleukin-10 and interleukin-1 receptor antagonist in the lung fluids of patients with the adult respiratory distress syndrome. Ann Intern Med 125: 191-196, 1996.

49. Lo CJ, Fu M and Cryer HG: Interleukin 10 inhibits alveolar macrophage production of inflammatory mediators involved in adult respiratory distress syndrome. J Surg Res 79: 179-184, 1998.

50. Peng XD, Dai LL, Huang CQ, He CM, Yang B and Chen LJ: Relationship between anti-fibrotic effect of Panax notoginseng saponins and serum cytokines in rat hepatic fibrosis. Biochem Biophys Res Commun 388: 31-34, 2009.

51. Lee JS, Choi HS, Kang SW, et al: Therapeutic effect of Korean red ginseng on inflammatory cytokines in rats with focal cerebral ischemia/reperfusion injury. Am J Chin Med 39: 83-94, 2011. 